\title{
OPEN AND DISTANCE LEARNING (ODL): STUDENTS ARE NO MORE JUST LEARNERS
}

\author{
Aisha Ismail \\ Department of Management Sciences, \\ Virtual University of Pakistan, \\ Pakistan \\ Email: ishaismail.gcu@gmail.com

\section{Sadaf Choudhary,} \\ Department of Management Sciences, \\ Virtual University of Pakistan, \\ Pakistan

\section{Rahila Hanif} \\ Department of Management Sciences, \\ Virtual University of Pakistan, \\ Pakistan
}

\begin{abstract}
The presence of diverse online resources in different delivery modes and the introduction of blended learning have posed new challenge for educational institutions to choose the method of learning which is not only effective but also a synchronized one. Changing a-synchronized contents into synchronized require an integrated effort from all members of e-learning system in the form of learning ecosystem. Learning ecosystem includes all components (content provider, infrastructure and consultant) that are necessary for the e-learning. In a learning system teacher are considered as content provider while students are acting as learners. A learning ecosystem aims to involve all stakeholders for the benefit of each other and does not restrict the role of students as learners only. Professional students enrolled in different degree programs have professional experience and are the source of asynchronous contents that can be synchronized through involving them as content vendors in learning ecosystem. This paper aims to introduce role of students as content vendors in the form of their professional experience from different industries. Their experiences will act as resources of educational institutions that can be assembled and used for awareness about business community among students. In order to integrate this valuable experiential knowledge, this paper will
\end{abstract}


propose a model for institutions. Model proposed by this study will change the existing role of students solely as leaner to the knowledge providers. This attained knowledge will also cover the existing gap between theory and application of knowledge.

\section{KEYWORDS}

Open and Distance learning, Ecosystem, E-learning, Content vendors

\section{INTRODUCTION}

Technological advancements in education system have not only provided different learning sources but it has also increased the distributed learning. This distributed knowledge at different networks requires synchronization for collective learning and creation of new knowledge (Nardi, Whittaker, \& Schwarz, 2000; Paavola \& Hakkarainen, 2005). A learning ecosystem can provide an ideal environment for collective learning of students and teachers through open learning and sharing of knowledge. In today's technologyoriented world of learning, e-learning institutions need a system that can help them in a collective learning similar to a natural ecosystem that work for mutual benefit and where resources are consumed and developed at the same time. Learning ecosystem can help to achieve this objective as e-learning institutions are taking learning environment as a self-sustainable learning system that provides tools and context to the learners to achieve their objectives i.e. through e-learning ecosystem (Pappas, 2005).

The concept of e-learning ecosystem is considered as second generation of elearning by research community (Dong, Zheng, Yang, Li, \& Qiao, 2009) a new model of holistic learning that requires practical implementation (Nguyen \& Hung, 2013). Studies on learning or e-learning ecosystem have identified four main components of e-learning ecosystem i.e. learners, learning content, learning context and learning infrastructure. In this model of e-learning ecosystem the role of students has been defined as "learners" or the learning actors. Depending upon the source of knowledge, learning can be formal or informal occurring in the classrooms, either through live discussions (synchronously) or by asynchronous means (Uden, Wangsa, \& Damiani, 2007). Professional students in ODL system are experiencing both synchronous and asynchronous learning. Along with academic learning, they are getting professional experiences through their jobs. They become content providers for the ODL institutions in terms of industrial knowledge through their professional 
experience. Students from different industries possess diverse experiences that need to be synchronized to become a knowledge resource for an effective elearning ecosystem. An effective e-learning ecosystem is possible if all members of the system interact with each other for mutual benefit and collective learning.

In an ecosystem participant are not just the consumers, they can be producers as well because they are interacting with each other for mutual benefit. Similarly, in e-learning ecosystem there is need to redefine the role of students as content providers, their role is not limited to content consumers they can contribute for collective learning in an e-learning ecosystem. Educational stakeholders have to think in a new way if they want vibrant e-learning ecosystem with an aim of mutual benefit, a change in structure, culture and the roles of members is required (Prince, Saveri, \& Swanson, September 2015). Considering this gap in the literature on e-learning ecosystem, this study aims to propose a new model with the additional role of students as content providers that can help in meeting the objectives of an e-learning ecosystem in true sense i.e. a holistic learning with mutual benefits. Identification of this new role of learners can increase the number of learners in e-learning ecosystem. This redefinition of learner's role will also change the role of teachers from content provider to content consumers (evaluating the contributions of learners i.e. at assessment stage) and for ODL institutions this can become a resource (by creating repository of student professional experiences) that can be used in adding practicality to the business education.

\section{LITERATURE REVIEW}

Distance learning is an important pillar of human learning ecosystem that involves construction, analysis, dissemination and recycling of learning resources among participants of leaning ecosystem i.e. educators, educates, administrators, society and policy makers. The use of technology has enabled e-leaning system to interact and share information through different learning activities similar to a natural ecosystem that is characterized with interconnected food chains. Web chains in e-learning create an interconnected system of information flow in e-learning systems (Vive, 2012).

A natural ecosystem is the system of organism (living and non-living) interacting with each other and with the environment, Uden, Wangsa, \& Damiani (2007) defined natural ecosystem as a dynamic and complex 
functional unit of organism, that interact with each other as well as with its environment. Learning ecosystem is a holistic system, an integration of four learning actors, learning-context, learning-contents and infrastructure i.e. learning-technology that provides an environment for the development of learners (individuals or groups) and the networks (Hung, 2014) supporting sharing and creation of new knowledge within and outside ecosystem (Hung, 2016). Nguyen and Hung (2013) elaborated e-learning ecosystem as a connected system of learning-subjects, learning-context, learning-contents, and learning-technologies; all components used to implement e-learning solutions(Uden, Wangsa, \& Damiani, 2007). E-Learning ecosystem constitutes technology i.e. e-learning infrastructure and knowledge resources available for the members of ecosystem for collective learning (Guild, 2013).

Main components of e-learning ecosystem are the actual organism (learners and the facilitators), learning system, resources and e-learning culture (Pappas, 2015), content provider, consultants and e-learning infrastructure (Uden Uden, Wangsa, \& Damiani, 2007), and content consumers, are the main contributors of a blended learning ecosystem (Detsis, Chalkias, \& Lasaridi, 2012; Nikolaidou et al., 2009). Nguyen and Hung (2013) have categorized elements of e-learning ecosystem under learning-subjects, contents, context and infrastructure.

In an ecosystem all the elements are interconnected and work as community benefiting each other. This integration of subjects, contents, context and learning technologies is required for getting optimum benefit and to use elearning ecosystem in a holistic manner (Uden Uden, Wangsa, \& Damiani, 2007). The organisms of the community can act as consumer and producer of food at the same time i.e. the system works through food chains or food webs. All members of ecosystem have to perform their roles in order to flourish in the ecosystem, moreover, a balance among all components of ecosystem is also required for mutual benefit of community as whole (Pappas, 2015). Similarly, in a learning ecosystem contribution from every member is necessary along with the resource consumption that is possible if learners also become the resource creator i.e. learners acting as knowledge resource. Considering the structure and functioning of an ecosystem connectivity is an important element for the effective working of all components of ecosystem and this connectivity is also a critical part in e-learning ecosystem. Learning activity in an e-learning ecosystem is term as networked learning or the learning through specialized 
nodes i.e. knowledge resources (Siemens, 2004). This connectivity is also crucial for ODL institutions because technological advancements and rapid growth of online resources has increased distributed knowledge that is termed as connective knowledge (Downes, 2006). This connective knowledge created during student-teacher interaction or by interaction of community members in an ecosystem is a new learning content in the e-learning ecosystem (Hung, 2014).

Literature on e-learning system has defined role of students as learners in the ecosystem but e-learning ecosystems are about sharing and creating knowledge and exchanging the experiences among the members of that system (Hung, 2016). It shows role of members of e-learning ecosystem is not just restricted to consumption, production or support rather contribution from every member is required for the proper functioning of an ecosystem.

Learning subject system in an e-learning ecosystem signifies the importance of learner's participation as content provider and peer level learning in the learning process(Nguyen \& Hung, 2013). Barab and Roth (2006) discussed the learning aspect of an ecosystem that is not about just scoring rather it is about successful participation in the learning system by increasing possibilities of action in the world. Learners role in e-learning ecosystem is not restricted to just consumption of knowledge resources and to get scores rather they can participate in creating other resources that can benefit others in the ecosystem.

The evolution of e-learning ecosystem has shifted the traditional concept of learning system i.e. the way learning takes place in an education system into a holistic learning that empower participants of education system in achieving more (Society, 2015) and identifying more opportunities to learn and share knowledge. This shift of learning system towards ecosystem can create diverse roles for the learners and educators and can increase the skills for learningsubjects to operate in the challenging environment (Prince et al., September 2015). Being the second generation of E-learning, the e-learning ecosystem is an emerging concept that is and its structure is still at a stage of infancy. The studies proposing models of e-learning ecosystem has defined four main components among which students are the important ones. Students have been defined as learners, the content consumers and the aspects of their contribution as content providers have not been addressed. This study aims to propose a model of e-learning ecosystem with an additional role of students as content- 
vendors that is necessary for the collective learning and will help in making the learning more interactive and worth sharing.

\section{OBJECTIVES}

1. To identify the new role of students as content providers

2. To propose a model of e-learning ecosystem for ODL institutions to identify students as content providers

\section{RESEARCH METHODOLOGY}

Based on the existing literature on learning and e-learning ecosystem this study has identified research gap in the proposed models of learning ecosystem. For conceptual model of e-learning ecosystem, relevant and accessible literature has been reviewed regardless of any time period. In this study, Hermeneutic approach that is recommended to interpret the text (Philip, 2011) is used to interpret the existing literature on e-learning ecosystem, learning ecosystem and blended learning ecosystem.

Secondary data has been consulted to identify the trend of student profiles (professions, working experiences, job positions etc.) to get an insight on the content enrichment that can be gained from students as content providers.

\section{Proposition}

Based on the literature discussed above this study proposes the following proposition to be either accepted or rejected: Proposition: Students can be content providers in the e-learning ecosystem.

\section{DISCUSSION}

Technological advancements in the field of education have made learning as networked learning that can take place anywhere and at any time. Learners in ODL institutions are experiencing both synchronous and asynchronous learning; they now possess more resources and more options of learning with flexibility and ease. This increase in number of resources has created distributed knowledge that needs synchronization for the benefit of educational community. Interaction among the participants of a learning system is a key to its success and for creating the collective and effective learning. Along with the shift in traditional learning system, e-learning is also experiencing a change. Change in the conceptions about learning system now it is not just an e-learning system it is becoming an e-learning ecosystem. Ecosystem is a combination of 
living and non-living organisms that are interacting with each other as well as with their environment for the mutual benefits. Similarly, e-learning institutions require an integrated system that work for the benefit of whole community. Members of an ecosystem are not isolated they are dependent on each other and they have to contribute something to their community for a sustainable ecosystem. In the same manner e-learning ecosystem also requires an integrated effort from all members including learners, educators, consultants etc. This introduction of e-learning ecosystem in the ODL is considered as second generation of e-learning and the structure of this learning system is at its infancy stage.

Literature on e-learning ecosystem has identified three main components of an e-learning ecosystem i.e. content providers, content consumers, and learning infrastructure. Students are tagged as learners and the resource consumers while teachers are the content providers or the facilitators in the e-learning ecosystem. Learning infrastructure provides support for the interaction of students and teachers. Following is the traditional model is proposed by Uden, Wangsa, \& Damiani (2007).

E-learning ecosystem proposed by (Uden et al., 2007)

Figure 1

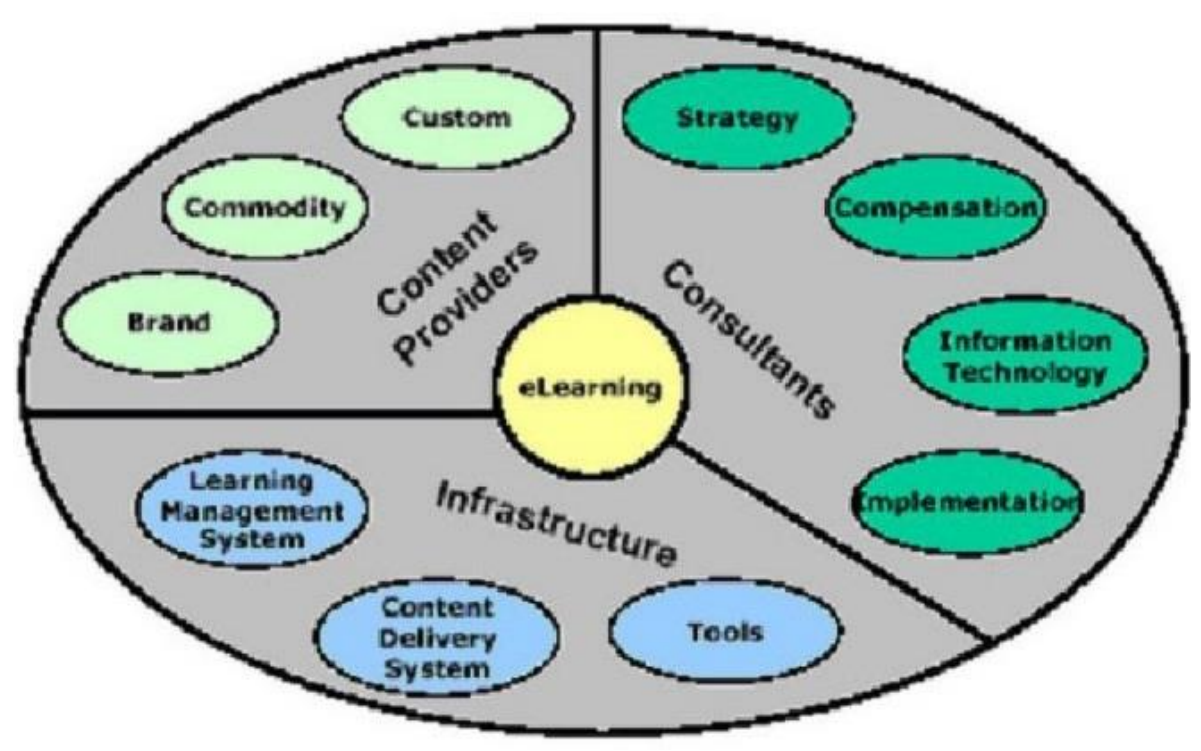


Figure 1 represents the model of e-learning ecosystem has discussed the role of students as learners while teachers are the content providers. The learning infrastructure that includes support in the form of technology, learning management systems, content delivery system and the tools for assessment is used to connect other elements of this learning model.

Considering the natural phenomenon in an ecosystem member of the system have to contribute something it means the role of students is not just to consume knowledge resources and to get grades but they can be contributors in the learning system by creating knowledge. This role of students as resource creators is missing in the proposed model of e-learning ecosystem. Taking into account this missing role of professional students as content providers in the form of sharing their professional experiences the proposed model of this study is as follows:

\section{Proposed Model with changing role of Students as content providers (OWN)}

Figure 2

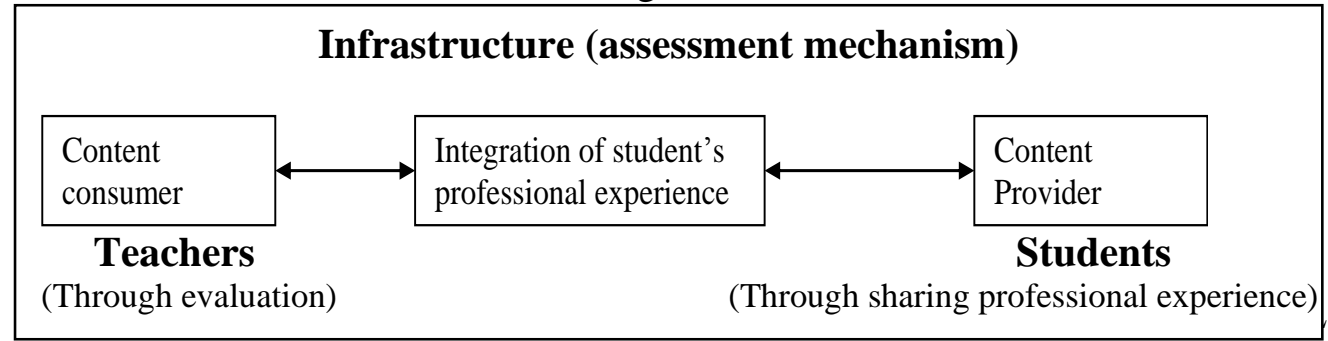

Secondary data on students' profile has identified that most of students in ODL institutions are professional and having diverse professional experiences that can be utilized in resource generation for reuse in the learning ecosystem. ODL students are dispersed geographically and have diversified knowledge and experiences, students being learners can also act as nodes in learning system as they share and can create new knowledge for other participants of ecosystem. Similar to a natural ecosystem, students in an e-learning ecosystem can consume and produce the knowledge at the same time initiating a collective learning. They can act as knowledge resource for the members of ecosystem by sharing their professional experiences. This study has proposed a new model (Figure 2) of e-learning ecosystem by adding the role of students as content providers. Professional students of ODL institutions will become knowledge 
resource for the learning system by sharing their professional knowledge that will be evaluated and utilized to add practical knowledge of industry for the students. ODL institutions will make a repository of such professional experiences (in audio or video from) that can be used as knowledge resource for other students and for the institutions in developing short courses or tutorials for the students to give them insight about industry working and the practices in different organizations. Learning infrastructure (medium of communication, LMS, the assessment tools etc.) will help students to perform their new role and to create an environment of collective learning for the mutual benefit of all members of ecosystem.

Following will be new model (Figure 3) of e-learning ecosystem after integrating the new aspect of students' role in the already existing model of elearning ecosystem:

Figure 3

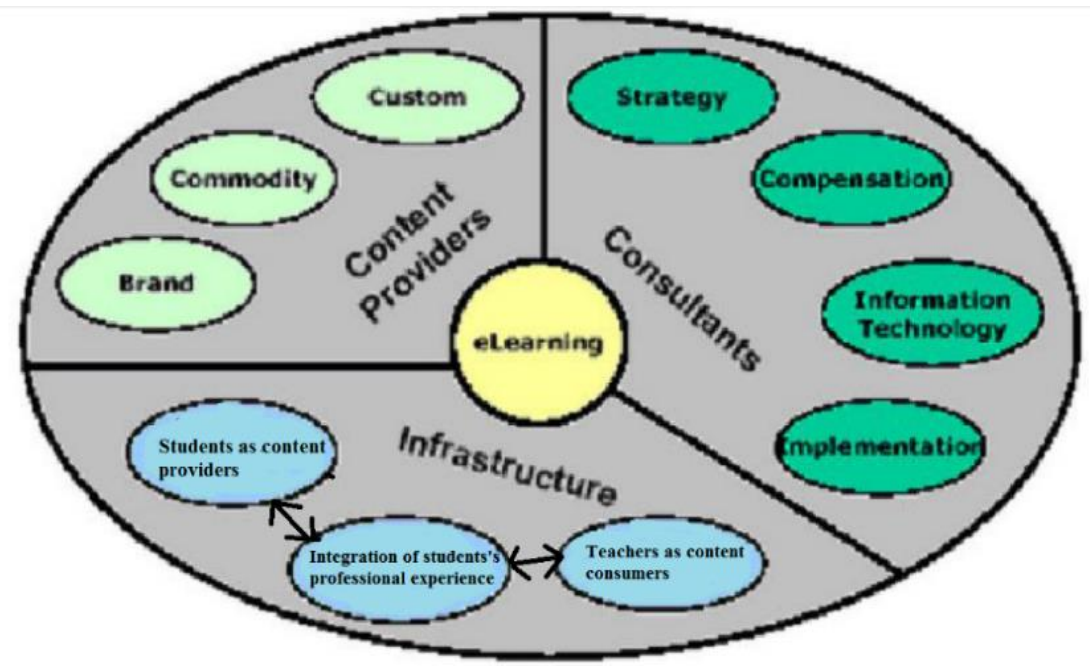

How students will become content provider in the e-learning ecosystem? Secondary data on profiles of students of higher education reveled that ODL institutions has significant number of executive students having professional experience of different industries that can be used as knowledge resource in the following way:

It will be mandatory for executive students to provide a video or audio 
recording on brief narration about their professional experience incorporating their industry type, job position, job description, and organizational culture. (making students a resource provider through sharing their experiences).

This provided resource (audio or video of professional experience) will be evaluated by the teacher on certain parameters (this will make teachers as resource consumers). Afterwards this will become the part of university's database after recommended editing (making Institutions as resource consumers). This repository can be used to give other students an overview of business world, how actually organizations are working and will add the practicality to the contents of the ODL institutions. This will also help in creating academia-industry alliance that is a missing link in the learning system.

The proposed model needs empirical testing to see its implication for learners, teachers, and policy makers and for the overall benefit of e-learning ecosystem.

\section{RECOMMENDATIONS}

Based on existing relevant literature on learning ecosystem, this study has identified new role of students' i.e. content providers that was tagged with the teachers in all previous models proposed for e-learning ecosystem. On the basis of this new role a modified model of e-learning system has been proposed that includes students as content consumers as well as content providers. Students will become the resource providers by sharing their professional experiences in a mandatory exercise (audio or video recording) that will be evaluated by the teachers and afterwards it will become part of institutional resource. This shared professional experience will help in revealing industry working and organizational practices for the knowledge of other students as well as for the ODL institutions to enrich their contents by adding practical knowledge, therefore accepting the proposition of the study to take students as content providers. Institutions can offer short courses or can add tutorials to existing subjects to enhance the effectiveness of the study programs. This data base of professional knowledge shared by executive students will also help in developing academia-industry alliance that is a missing link in the learning system.

This proposed e-learning model and the identification of new role of learners as content-providers or content vendors need empirical testing to verify its implications for students, educators and the policy makers. This study has 
opened a new horizon for ODL researchers, this is just a stepping stone and the sky is open for further study.

\section{REFERENCES}

Barab, S. A., \& Roth, W.-M. (2006). Curriculum-based ecosystems: Supporting knowing from an ecological perspective. Educational Researcher, 35(5), 313.

Detsis, M. N. V., Chalkias, C. S. C., \& Lasaridi, K. (2012). The blended learning ecosystem of an academic institution in Greece. Evaluating the Impact of Technology on Learning, Teaching, and Designing Curriculum: Emerging Trends: Emerging Trends, 173.

Dong, B., Zheng, Q., Yang, J., Li, H., \& Qiao, M. (2009). An e-learning ecosystem based on cloud computing infrastructure. Paper presented at the Advanced Learning Technologies, 2009. ICALT 2009. Ninth IEEE International Conference on.

Downes, S. (2006). Learning networks and connective knowledge. Collective intelligence and elearning, 20, 1-26.

Guild, T. e. (2013). What is a Learning Ecosystem? , from http://twist.elearningguild.net/2013/11/what-is-a-learning-ecosystem/

Hung, N. M. (2014). Using ideas from connectivism for designing new learning models in Vietnam. in International Journal of Information and Education Technology.

Hung, N. M. (2016). On Semantic Model of Formative Assessment in Connection with Learning Ecosystem. International Journal of Information and Education Technology, 6(1), 54.

Nardi, B., Whittaker, S., \& Schwarz, H. (2000). It's Not What You Know, It's Who You Know: Work in the Information Age. : , 5 (5). First Monday:Peerreviewed Journal on the Internet, 5(5).

Nguyen, N. H., \& Hung, N. M. (2013). On the procedural structure of learning ecosystem toward competency learning model. Available at SSRN 2481764.

Nikolaidou, M., Sofianopoulou, C., Alexopoulou, N., Abeliotis, K., Detsis, V., Chalkias, C., Anagnostopoulos, D. (2009). EXPLORING A BLENDED LEARNING ECOSYSTEM IN THE ACADEMIC ENVIRONMENT.

Paavola, S., \& Hakkarainen, K. (2005). The knowledge creation metaphor-An emergent epistemological approach to learning. Science \& Education, 14(6), 535-557. 
Pappas, C. (2015). The eLearning Ecosystem Metaphor: Key Characteristics And Basic Components, from http://elearningindustry.com/the-elearningecosystem-metaphor-key-characteristics-and-basic-components

Prince, K., Saveri, A., \& Swanson, J. (September 2015). Exploring the Future Education Workforce: New Roles for an Expanding Learning Ecosystem. Knowledge Works.

Siemens, G. (2004). Connectivism: A learning theory for the digital age. International Journal of Instructional Technology and Distance Learning: online], http://www. elearnspace. org/Articles/connectivism. htm.

Society, T. A. I. C. (2015). INTRODUCTION - The Learning Ecosystem

Uden, L., Wangsa, T., \& Damiani, E. (2007). The future of E-learning: E-learning ecosystem. Paper presented at the Digital EcoSystems and Technologies Conference, 2007. DEST'07. Inaugural IEEE-IES.

Vive, K. (2012). System and Technology Advancements in Distance Learning: Information Science Reference. 